\section{Fasciculus Gracilis}

John E. Mendoza

Department of Psychiatry and Neuroscience, Tulane Medical School and SE Louisiana Veterans Healthcare System, New Orleans, LA, USA

\section{Definition}

Fiber tract consisting of axons from the dorsal nerve roots that mediate fine tactile discrimination (stereognosis), proprioception, and vibration sense for the lower extremities and lower portion of the trunk. Along with the fasciculus cuneatus, which carries similar sensory information from the upper extremities and trunk, these pathways constitute the posterior (dorsal) columns of the spinal cord. Both are ipsilateral pathways (sensory information comes from the same side of the body as the tracts themselves). The fasciculus gracilis synapses in the nucleus gracilis in the medulla, where secondary fibers eventually cross the midline forming the medial lemniscus which terminates in the thalamus. Spinal cord lesions involving this pathway would result in disturbances in position sense, vibration, two-point discrimination, or other stereognostic abilities such as being able to discriminate numbers traced on the skin in the ipsilateral lower extremity.

\section{Cross-References}

Dorsal Nerve Roots

- Posterior Columns

\section{References and Readings}

Mendoza, J. E., \& Foundas, A. L. (2008). The somatosensory systems. In J. E. Mendoza \& A. L. Foundas (Eds.), Clinical neuroanatomy - A neurobehavioral approach (pp. 23-47). New York: Springer. 